\title{
Very Low Threshold InGaAsP Mesa Laser
}

\author{
T. R. CHEN, L. C. CHIU, K. L. YU, U. KOREN, A. HASSON, S. MARGALIT, AND A. YARIV
}

\begin{abstract}
Very low threshold currents InGaAsP/InP terrace mesa (T-ME) lasers with an unpassivated surface have been fabricated on semi-insulating (SI) InP substrates. Fabrication of the lasers involves a single-step liquid phase epitaxial (LPE) growth and a simple etching process. Lasers operating in the fundamental transverse mode with threshold currents as low as $6.3 \mathrm{~mA}$ (for a cavity length of $250 \mu \mathrm{m}$ ) have been obtained. Comparison between the unpassivated lasers and those passivated using the mass transport technique is described.
\end{abstract}

I $\mathrm{T}$ has long been suspected that in semiconductor lasers with buried active regions there exists a substantial amount of leakage current bypassing the active region junction through the cladding burying layers. Such a leakage would give rise to an undesirable increase in threshold and possibly to a reduced quantum efficiency. In the case of InGaAsP/InP buried heterostructure lasers, there have been experimental indications of such leakage currents despite the presence of a reverse biased blocking junction [1]-[3] .

We were thus led to experiment with a structure in which this leakage current could be eliminated. A laser structure in which this leakage was minimized and with threshold currents in the range of $40-50 \mathrm{~mA}$ already showed encouraging results [4]. The relatively benign nature of the quaternary-air interface, in terms of nonradiative recombination, as evidenced by the slow facet degradation rate [5], [6] and possibly low surface recombination velocity [7], held some promise that very low threshold lasers whose performance would not be strongly degraded by this interface could be realized. This idea was successfully applied and tested in a new structure in which the active region was bounded transversely on the sides by SI InP and air, respectively. Thus, current leakage was virtually eliminated.

As a result, very low threshold current lasers were successfully fabricated with a single-step liquid phase epitaxial (LPE) growth and a simple etching process. A schematic drawing of the structure is shown in Fig. 1(a) and (b). Using $\mathrm{Si}_{3} \mathrm{~N}_{4}$ as etching mask, the substrate was first selectively etched down along the (011) crystallographic direction, giving rise to a terrace shape. Three LPE layers, $\mathrm{n}^{+}-\mathrm{InP}$, undoped InGaAsP, and $\mathrm{p}$-InP, were grown successively on the etched bottom region [Fig. 1(a)]. After the LPE growth, a new $\mathrm{Si}_{3} \mathrm{~N}_{4}$ etching mask was deposited, and windows for chemical etching were opened with one side parallel to and approximately $5 \mu \mathrm{m}$ away from the edge of the terrace. The p-InP cladding layer was selec-

Manuscript received September 7, 1982; revised November 19, 1982. This work was supported by the National Science Foundation, the U.S. Office of Naval Research, and the U.S. Air Force Office of Scientific Research.

The authors are with the California Institute of Technology, Pasadena, CA 91125.

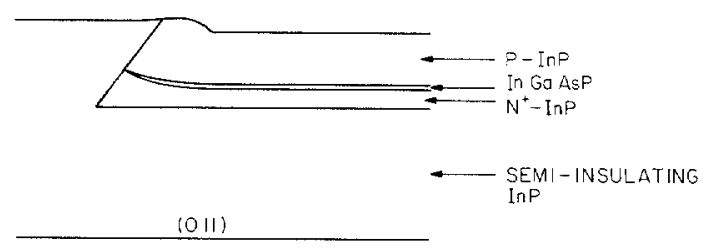

(a)
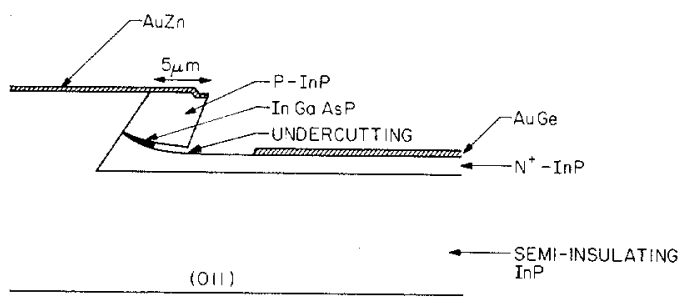

(b)

Fig. 1. (a) Schematic cross section of the three basic layers of the T-ME laser on semi-insulating InP along the (011) direction. (b) Schematic cross section of the fabricated T-ME laser.

tively removed with a solution of hydrochloric acid and water. The $\mathrm{Si}_{3} \mathrm{~N}_{4}$ mask was then removed and the wafer was cleaned. The exposed quaternary layer was then etched with a solution of $\mathrm{KOH}: \mathrm{K}_{3} \mathrm{Fe}(\mathrm{CN})_{6}: \mathrm{H}_{2} \mathrm{O}$. For this structure, a certain amount of undercutting into the InGaAsP layer was intended to obtain narrow active layer width. Since one side of the active region is bounded by the SI terrace wall, etching of the active layer proceeds from the exposed side only, and the etching process is thus quite controllable. Consequently, very narrow active layers with uniform widths could be realized. This is essential to obtaining high quality, low threshold lasers. After etching, the wafer was cleaned with deionized water, and gold alloys were deposited for contacts. A schematic representation of the processed laser is shown in Fig. 1(b).

Since the etching and undercutting of the quaternary layer was performed from one side only, the overhanging top layer was connected to and supported by the SI substrate. This resulted in structurally stable devices, and no problems with the collapse of the top layer were encountered. By undercutting into the quaternary layer to obtain narrow active layer width, rather than etching vertically down very near the terrace wall without undercutting [1], the area available for making electrical contacts is broader and presents no processing problems [Fig. 1(b)]. In addition, this allows direct comparison to identical lasers passivated with mass transport technique.

For active regions with a volume of $\sim 0.2 \times \sim 1.5-2 \times 250$ $\mu \mathrm{m}^{3}$, the average threshold current was about $14 \mathrm{~mA}$. The lowest obtained was $6.3 \mathrm{~mA}$, which is comparable to the lowest threshold quaternary lasers reported in literature to date. A typical light versus current characteristic is shown in 


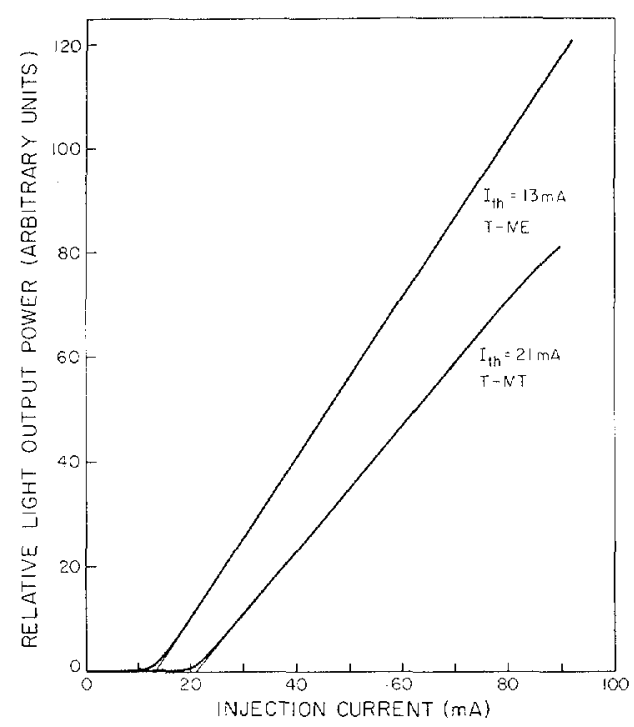

Fig. 2. $L-I$ characteristic of a T-ME laser. The $L-I$ characteristic of a T-MT laser is also shown for comparison.

Fig. 2. The laser exhibits excellent linearity exceeding seven times the threshold current. Differential quantum efficiency is about 10-20 percent higher than for the terrace mass transport (T-MT) lasers [8].

Since the active region is "buried" on the sides by SI InP and air, respectively, there exists negligible current leakage. Despite the exposure of one side to air, very low threshold current was obtained. This indicates that surface recombination velocity in InGaAsP is indeed low, and is also consistent with the much slower facet degradation rate of InGaAsP/InP system as compared to GaAs/AlGaAs system [5], [6]. Although the lasers in [4] also possessed an unpassivated surface, unfortunately, the wider width $(\sim 6 \mu \mathrm{m})$ and much higher threshold currents (40-50 mA) could not lend direct evidence to the low surface recombination velocity. It should nevertheless be noted that the exact nature of the unpassivated InGaAsPair interface is still unclear.

To ascertain the role and effects of leakage current and surface passivation on the performance of lasers in which the active region is buried in InP, we have fabricated lasers identical in all respects to that described above, except that the air space next to the active region was "plugged" by epitaxial InP using the mass transport technique [8], [9]. Mass transport of InP was achieved using an InP cover wafer and heating at $\sim 675^{\circ} \mathrm{C}$ for about $45 \mathrm{~min}$. An SEM photograph of the resulting terrace mass transport laser (T-MT) is shown in Fig. 3. The T-MT lasers with similar dimensions had threshold currents averaging around $20 \mathrm{~mA}$, compared to $14 \mathrm{~mA}$ in the case of the air interface T-ME's. The average $L-I$ characteristics of T-MT's are linear up to $4 I_{\mathrm{th}}$. The corresponding figure for the T-ME's is $7 I_{\mathrm{th}}$. On the average, the $T_{o}$ of T-MT's falls in the range of $40-50 \mathrm{~K}$. For the T-ME's, the $T_{o}$ lies between $60-70 \mathrm{~K}$. The far-field patterns are shown in Fig. 4. Here the scattering from the exposed sidewall of the T-ME's is apparent. However, the patterns are stable with increasing injection current. The doping concentrations of the active layers estimated from $\mathrm{C}-\mathrm{V}$ measurements were $5.0 \times 10^{17} \pm 1.0 \times 10^{17} \mathrm{~cm}^{-3}$ for T-ME's and $1.5 \times 10^{18} \pm 0.5 \times 10^{18} \mathrm{~cm}^{-3}[10]$ for T-MT's.

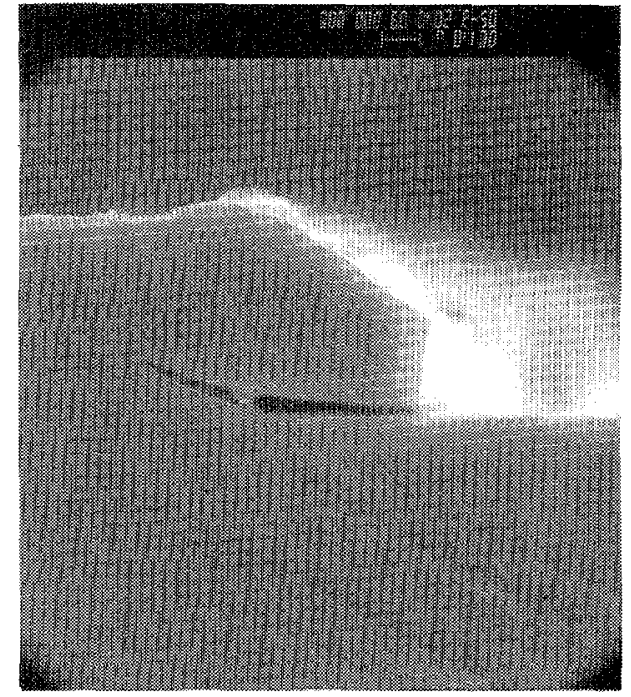

Fig. 3. An SEM photograph of the T-MT laser after mass transport. The T-ME laser would appear to be identical except for the absence of the mass transported region.

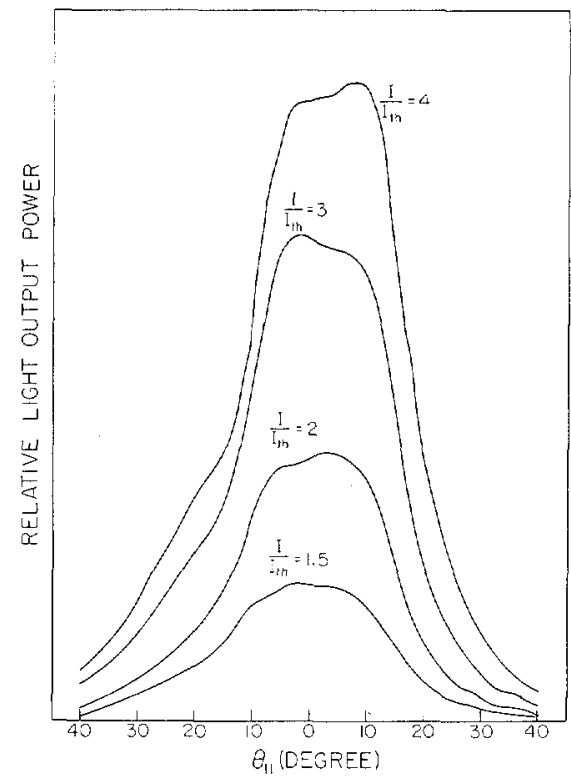

(a)

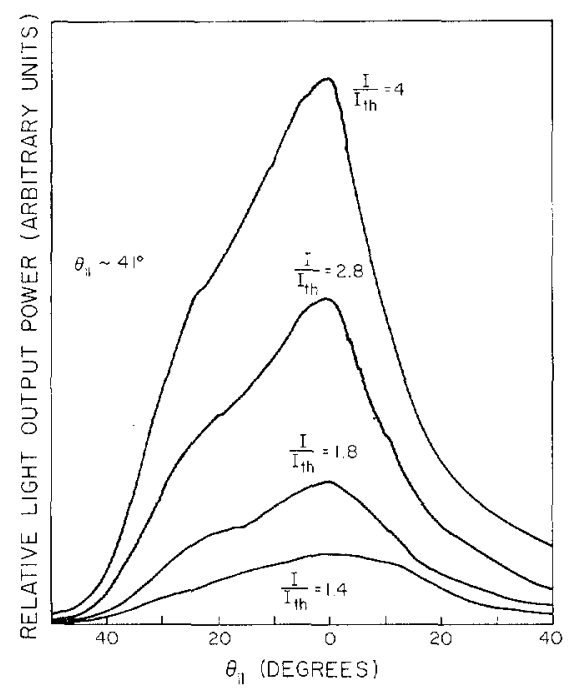

(b)

Fig. 4. Far-field patterns of the (a) T-MT and (b) T-ME lasers. 

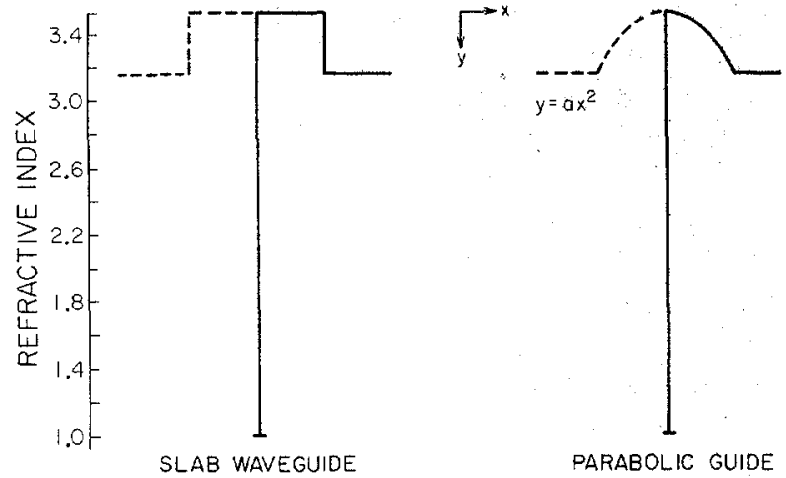

Fig. 5. Slab and parabolic waveguides used in the estimation of the cutoff width.

These results are further supported by the measured reverse breakdown voltages of the devices. The higher doping in T-MT's may be a result of $\mathrm{Zn}$ diffusion at the temperature of $675^{\circ} \mathrm{C}$ during the mass transport process [11]. Apart from the far-field pattern, the improved performance of the T-ME's could possibly be attributed to the negligible current leakage. However, the effect of the leakage current on the $T_{o}$ depends on the temperature dependence of the shunt resistance of the leakage path [3]. Thus, the exact nature of the higher $T_{0}$ of the T-ME's as compared to the T-MT's is not certain. We recognize that the lower doping concentrations in the active layers of the T-ME's may be a contributing factor. It should be stressed that possible long term effects of the unpassivated surface on the performance and reliability of the T-ME's remain unknown. In this context, the newly discovered [9] mass transport technique does provide an excellent way to protect the surface.

No lasing action was observed on a wafer where the active layer was etched to a very narrow width $(<\sim 0.5 \mu \mathrm{m})$, despite the presence of a typical InGaAsP-InP p-n junction. It is interesting to point out that in contrast to the T-MT's, there exists a cutoff width of the waveguide for the T-ME structure. Since the index step between the InGaAsP active layer and the InP cladding layer is $\sim 0.36$ [12], and the step between the air and the quaternary layer is much larger, the modes of the T-ME structure can be approximated to a high degree of accuracy by the odd TE modes of a guide with twice the width (Fig. 5). Using values of refractive indexes from [12], the cutoff width is estimated approximately for both the slab and parabolic guides [13]. The results are shown in Table I. An interesting feature of these asymmetric waveguides, besides the existence of a cutoff width, is that the cutoff widths for the higher order modes are much larger than those in the corresponding symmetric guides. As can been seen from Table I, for a slab waveguide, there is a factor of 3 in width at which first order modes can propagate. Therefore, wider active regions could be tolerated without lasing in higher order transverse modes.

In conclusion, very low threshold InGaAsP/InP lasers with
TABLE I

Estimated Cutoff Widths (IN $\mu \mathrm{m}$ ) for the Fundamental and First Order Modes of the Slab and Parabolic Waveguides as Shown in Fig 5. For the Parabolic Case, $y=a x^{2}$ Is Assumed for the Geometrical Shape of the Guide with Values of $a\left(\mu \mathrm{m}^{-1}\right)$ as Specified.

\begin{tabular}{|c|c|cc|}
\hline N & Slab & \multicolumn{2}{|c|}{ Parabolic } \\
N & Cutor Widtb $(\mu \mathrm{m})$ & $\left(\mu \mathrm{m}^{-1}\right)$ & Cutor Width $(\mu \mathrm{m})$ \\
\hline Fundamental & 0.16 & 50 & .16 \\
- & - & 50 & .17 \\
- & - & 30 & .18 \\
- & - & 20 & .20 \\
\hline First order & .48 & 50 & 48 \\
- & - & 40 & .52 \\
- & - & 30 & .54 \\
- & - & 20 & .60 \\
\hline
\end{tabular}

an unpassivated surface have been fabricated on SI substrate with a single LPE and an etching process, indicating a low sur. face recombination velocity at the unpassivated InGaAsP-air interface. This provides a simple way to fabricate very low threshold quaternary lasers. Comparison of this laser with the mass transport lasers revealed certain interesting and important factors affecting the laser performance.

\section{REFERENCES}

[1] R. J. Nelson, R. B. Wilson, P. D. Wright, P. A. Barnes, and N. K Dutta, "CW electrooptical properties of InGaAsP $(\lambda=1.3 \mu \mathrm{m})$ buried-heterostructure lasers," IEEE J. Quantum Electron., vol. QE-17, pp. 202-207, Feb. 1981.

[2] P. D. Wright, W. B. Joyce, and D. C. Craft, "Electrical derivative characteristics of InGaAsP buried heterostructure lasers," $J$. Appl. Phys., vol. 53, pp. 1364-1372, Mar. 1982.

[3] N. K. Dutta, P. D. Wright, R. J. Nelson, R. B. Wilson, and P. R. Besomi, "InGaAsP laser with high $T_{o}$," IEEE $J$. Quantum Electron., vol. QE-18, pp. 1414-1416, Oct. 1982.

[4] T. Matsuoka, Y. Suzuki, Y. Noguchi, and H. Nagai, "GaInAsP/ InP DH laser on semi-insulating InP substrate with terrace structure," Electron. Lett., vol. 18, pp. 359-361, Apr. 1982.

[5] M. Morimoto, H. Imai, K. Hori, M. Takusagawa, and M. Fududa, "Facet degradation of InGaAsP/InP double heterostructure lasers," Appl. Phys. Lett., vol. 37, pp. 1082-1084, Dec. 1980.

[6] H. Temkin, S. Mahajan, M. A. DiGiuseppe, and A. G. Dentai, "Optically induced catastrophic degradation in InGaAsP/InP layers," Appl. Phys. Lett., vol. 40, pp. 562-565, Apr. 1982.

[7] L. C. Chiu, T. R. Chen, C. Harder, P. C. Chen, and A. Yariv, unpublished.

[8] T. R. Chen, L. C. Chiu, K. L. Yu, U. Koren, A. Hasson, S. Margalit, and A. Yariv, "Low threshold InGaAsP terrace mass transport laser on semi-insulating substrate," Appl. Phys. Lett., Dec. 1982.

[9] Z. L. Liau and J. N. Walpole, "A novel technique for GaInAsP/ InP buried heterostructure laser fabrication," Appl. Phys. Lett., vol. 40, pp. 568-570, Apr. 1982.

[10] These values represent the average taken over 6-8 diodes (of each) from the same wafer.

[11] C. B. Su, J. Schlafer, J. Manning, and R. Olshansky, "Measurement of radiative and Auger recombination rates in p-type InGaAsP diode lasers," Electron. Lett., pp. 595-596, July 1982.

[12] S. Adachi, "Refractive indices of III-V compounds: Key properties of InGaAsP relevant to device design," J. Appl. Phys., vol. 53, pp. 5863-5869, Aug. 1982.

[13] For the parabolic guides, both the refractive index and actual shape of the guide are taken to be parabolic, with the geometrical shape described by $y=a x^{2}$ with $a$ as a parameter [Table 1]. 